\title{
Análise de conhecimento sobre infecções sexualmente transmissíveis e uso de anticoncepcional por pacientes de uma Unidade Básica de Saúde da Capital do Estado de Rondônia
}

\author{
Sexually transmitted diseases knowledge analysis and contraceptive use by patients of a \\ Basic Health Unit in Rondônia State Capital \\ Análisis del conocimiento sobre infecciones de transmisión sexual y uso de \\ anticonceptivos por parte de pacientes en una Unidad Básica de Salud de la Capital del \\ Estado de Rondônia
}

Anitha de Cássia Ribeiro da Silva ${ }^{1 *}$, Luana Kamila Castilho Rodrigues ${ }^{1}$, Alana Pascoal de Almeida ${ }^{1}$, Felipe de Lima Sarah" ${ }^{1}$, Maria Clara Souza Brito ${ }^{1}$, Maria Paula Freire da Fonseca ${ }^{1}$, Lorrany Alonso Quenca $^{1}$, Gabriela Rocha Meira de Melo Soares ${ }^{1}$, Mariana Bezerra dos Santos ${ }^{1}$, Arlindo Gonzaga Branco Junior ${ }^{1,2}$.

\section{RESUMO}

Objetivo: Avaliar o perfil epidemiológico e nível de conhecimento sobre infecções sexualmente transmissíveis e uso de anticoncepcionais entre pacientes de uma Unidade Básica de Saúde da capital de Rondônia. Métodos: Estudo de caráter descritivo, exploratório e quantitativo realizado com mulheres de idade entre 18 a 40 anos, atendidas na Unidade Básica de Saúde. Composto por 15 questões sobre IST e uso de anticoncepcionais e avaliação do perfil epidemiológico. Resultados: Com base na análise dos dados descreve-se que a idade prevalente das usuárias da Unidade Básica de Saúde é de 18 a 21 anos correspondendo a $33 \%$ do total, nível de escolaridade predominou mulheres com ensino médio completo representado por $30 \%$, já a renda mensal prevalente o valor de 1 salário mínimo se sobressaiu em $75 \%$. Conclusão: Conforme o exposto verifica-se que a maioria das entrevistadas se encontram em plena faixa sexualmente ativa o que propicia e com ensino médio completo o que conforme a literatura contribui para um conhecimento mais específico sobre IST e métodos contraceptivos, no entanto, ainda é necessárias medidas de intervenção que contribuem para ampliar o conhecimento e assim contribuir para melhoria do uso de contraceptivos e compreender o processo de prevenção de IST.

Palavras-chave: Anticoncepção, Doenças Sexualmente Transmissíveis, Mulheres, Educação em saúde.

\begin{abstract}
Objective: To evaluate the epidemiological profile and level of knowledge about sexually transmitted infections and use of contraceptives among patients in a Basic Health Unit in the capital of Rondônia. Methods: A descriptive, exploratory and quantitative study carried out with women aged between 18 and 40 years, attended at the Basic Health Unit. Composed of 15 questions about STI and use of contraceptives and assessment of the epidemiological profile. Results: Based on the data analysis, it is described that the prevalent age of users of the Basic Health Unit is 18 to 21 years old, corresponding to $33 \%$ of the total, schooling level predominated women with complete high school represented by $30 \%$, already the prevailing monthly income, the value of 1 minimum wage stood out by $75 \%$. Conclusion: As shown above, it appears that most of the interviewees are in the full sexually active range, which provides and with complete high school, which according to the literature contributes for a more specific knowledge about STIs and contraceptive methods, however, intervention measures are still needed that contribute to expand knowledge and thus contribute to improving the use of contraceptives and understanding the STI prevention process.
\end{abstract}

Key words: Contraception, Sexually Transmitted Diseases, Women, Health education.

\footnotetext{
${ }^{1}$ Centro Universitário São Lucas (UniSL), Porto Velho - RO. *E-mail: anithadecassia@gmail.com

2 Universidade Federal de Rondônia, Porto Velho - RO.
}

SUBMETIDO EM: 2/2021

ACEITO EM: 2/2021

PUBLICADO EM: $3 / 2021$ 


\section{RESUMEN}

Objetivo: Evaluar el perfil epidemiológico y el nivel de conocimiento sobre infecciones de transmisión sexual y uso de anticonceptivos en pacientes de una Unidad Básica de Salud de la capital de Rondônia. Métodos: Estudio descriptivo, exploratorio y cuantitativo realizado con mujeres de entre 18 y 40 años, atendidas en la Unidad Básica de Salud. Compuesto por 15 preguntas sobre ITS y uso de anticonceptivos y evaluación del perfil epidemiológico. Resultados: Con base en el análisis de datos, se describe que la edad prevalente de los usuarios de la Unidad Básica de Salud es de 18 a 21 años, correspondiente al $33 \%$ del total, el nivel de escolaridad predominó mujeres con bachillerato completo representado por $30 \%$, Ya el ingreso mensual prevaleciente, el valor de 1 salario mínimo se destacó en $75 \%$. Conclusión: Como se muestra arriba, parece que la mayoría de los entrevistados se encuentran en el rango sexualmente activo completo, lo que proporciona y con la escuela secundaria completa, que de acuerdo con la literatura contribuye para un conocimiento más específico sobre las ITS y los métodos anticonceptivos, sin embargo, aún se necesitan medidas de intervención que contribuyan a ampliar el conocimiento y así contribuir a mejorar el uso de anticonceptivos y comprender el proceso de prevención de las ITS.

Palabras clave: Anticoncepción, Enfermedades de Transmisión Sexual, Mujeres, Educación en salud.

\section{INTRODUÇÃO}

As infecções sexualmente transmissíveis são um problema de saúde pública, que no ano de 2012 atingiu cerca de 357,4 milhões de casos novos, com prevalência na faixa etária de 15 a 49 anos, sendo a maioria dos casos em países em desenvolvimento (PINTO VM, et al., 2018). Com base no mesmo autor, não há dados que informem a amplitude de infeções no Brasil, visto que, a maioria não é de notificação compulsória, além da pobreza de estude sentinela de base populacional.

No entanto, há descrito que o número de parceiros, sexo desprotegido, uso de álcool e drogas ilícitas, bem como o tabagismo são considerados como comportamento de risco para ocorrência de Infecções Sexualmente Transmissíveis (IST) (NEVES RG, et al., 2017). A instalação de IST está diretamente relacionada a atividade sexual, no Brasil a literatura indica que a idade de início é por volta dos 13 anos de idade, sendo um padrão comum nos países em desenvolvimento que não possuem programa de educação sexual consolidado (LARA LAS e ABDO CHN, 2015).

Com o início da vida sexual precoce há mais chances de contrair IST tanto em indivíduos do sexo masculino quanto no feminino, além disso as taxas de gravidez também aumentam promovendo instabilidade no desenvolvimento social, pessoal, familiar e educacional (NEVES RG, et al., 2017). No contexto brasileiro, estudos apontam que a cada ano nascem mais de 14 milhões de crianças cujas mãe são adolescentes, sendo 18\% das adolescentes com pelo menos um filho no território nacional (RASMUSSEN VS, et al., 2011).

Sabe-se que no ano de 1983 fora implantado, no Brasil, o Programa de Assistência Integral à Saúde da Mulher (PAISM), o qual tinha como objetivo padronizar ações com atenção ao ciclo e etapas da vitalidade feminina, por meio de princípios de atenção preventiva, de cuidados curativos e, inclusive, do planejamento familiar (CARRENO I, et al., 2006).

Entretanto, apesar da teoria ser baseada no processo de regionalização desse programa a aplicação não ocorreu de forma efetiva em todo o território. Por meio de uma análise de fatos, percebeu-se um descomprometimento de cunho político com a execução eficaz do PAISM de acordo com estudos feitos por Osis MJMD (1994). Pode-se perceber o detrimento dos resultados do programa ao verificar que a estimativa de gestação indesejada por brasileiras é de 10 milhões, visto o uso inadequado ou o não uso de métodos anticoncepcionais (CURITIBA, 2002).

É válido, assim, ter conhecimento das causas, de forma generalizada, que levam à gravidez precoce, como por exemplo: acaso; ingenuidade; submissão; violência; dificuldade de obter algum método contraceptivo; dificuldade em negociar o uso do preservativo; forte desejo pela maternidade, expectativas de mudança de status social e de obtenção de autonomia; desejo de estabelecer uma união estável; crença; e, outros fatores de natureza objetiva ou subjetiva (RASMUSSEN VS, et al., 2011). 
A abordagem da introdução do método anticoncepcional na vida sexual do indivíduo deve levar em conta a idade, nível socioeconômico, paridade, contexto social e religioso. Além disso, a participação do parceiro na tomada da decisão é bastante incentivada para melhor aceitação do método e para melhor qualidade as informações sobre a saúde reprodutiva (CURITIBA, 2006).

Apesar de números indicarem que a prevalência do uso de métodos contraceptivos no Brasil é alta, estes resultados se concentram mais na laqueadura e na pílula anticoncepcional por $40 \%$ e $21 \%$, respectivamente, das mulheres adultas, essencialmente, deixando vulnerável à Infecções Sexualmente Transmissíveis (IST's) (RASMUSSEN VS, et al., 2011).

As IST até hoje geram significativa morbimortalidade no Brasil e no mundo. Agrupadas em bacterianas, virais e parasitárias, essas infecções estão presentes em mais de 190 milhões de prontuários (BRITTO AMA, et al., 2018). Dentre esses 190 milhões de casos, 40 milhões correspondem ao HIV e dentre os 40 milhões, mais de 630 mil pessoas acometidas são brasileiras, fazendo com que o Brasil seja responsável por compreender um terço dos indivíduos vivendo com HIV em toda américa latina (DEL BIANCO AAM, 2017).

A persistência de casos de HIV e outras infecções sexualmente transmissíveis em mulheres, decorre tanto de uma maior vulnerabilidade com relação a papéis sociais de gênero, desequilíbrio de poder entre homens e mulheres e violência contra mulher quanto de aspectos biológicos, econômicos e culturais (DUARTE MTC, et al., 2014).

As mulheres comprometidas por doenças sexualmente transmissíveis fazem parte de um grupo desafiador para a saúde pública brasileira. A elevada prevalência de casos entre mulheres jovens, a falta de diagnósticos precoces e a escassez de meios estratégicos para prevenção dessas doenças fazem com que as IST's sejam a segunda causa de morbidade entre mulheres jovens de países em desenvolvimento (LUPPI CG, et al., 2011).

A análise das características de mulheres brasileiras, especialmente as relacionadas a uma maior vulnerabilidade (como baixas condições socioeconômicas, mulheres em encarceramento, em situação de rua entre outras), é útil para a procura de meios e estratégias para uma diminuição da prevalência de acometimentos ginecológicos como as IST's e gravidezes precoces (DUARTE MTC, et al., 2014).

O acesso à educação sexual para, entre outras coisas, adquirir informações sobre prevenção de IST e gravidez precoce é imprescindível e impostergável. $O$ fato de jovens terem aulas sobre sexualidade não tem influência direta sobre a decisão do início da atividade sexual, mas sim, tem influência de forma consciente fazendo com que a atividade sexual seja iniciada de forma apropriada, diminuindo o número de gestações (SAITO MI e LEAL MM, 2000).

Com isso, a atuação dos profissionais de saúde é importante, tendo como principal foco contribuir para conscientização e mudança na visão da população acerca do tema, somado a isso, a promoção do atendimento imediato contribui para diminuição da vulnerabilidade e complicações decorrentes das IST, bem como o diagnóstico e tratamento ser realizado de modo rápido, sendo complementado com medidas educativas para com a saúde individual e coletiva (TERRA AAA e SILVA GA, 2017).

Diante desse cenário, esse estudo analisou o conhecimento de mulheres atendidas em uma Unidade Básica de Saúde da capital do Estado de Rondônia, sobre infecções sexualmente transmissíveis e uso de métodos contraceptivos para comparação de dados compreensão da realidade dessa parcela da população atendida, possibilitando assim a aplicação de medidas para contornar possíveis vulnerabilidade apresentada.

\section{MÉTODOS}

Estudo de caráter descritivo, exploratório e quantitativo realizado com mulheres de idade entre 18 a 40 anos, que foram atendidas em uma Unidade Básica de Saúde, localizada na cidade de Porto Velho, Rondônia. Foram convidadas a participar da pesquisa 55 mulheres, no entanto apenas 46 aceitaram responder o questionário e desse grupo 33 se enquadravam nos critérios de inclusão. A coleta de dados ocorreu durante duas semanas no período vespertino entre as 13 as 17 horas no horário de Porto Velho, Rondônia. 
Devido ao cenário decorrente da pandemia de COVID-19 no Estado de Rondônia e com base nos decretos emitidos pelo governo do Estado com o intuito de mitigar o contágio entre os indivíduos (RONDÔNIA, 2020), os auxiliares da pesquisa estavam paramentados com equipamentos de proteção individual, respeitando as orientações de distanciamento social, bem como fizeram a assepsia das canetas e pastas usadas como auxílio na coleta de dados, contribuindo para o controle da transmissão do COVID-19.

Para solicitação da participação na pesquisa, foi comunicado no que consistia o estudo, qual a finalidade, a segurança dos dados fornecidos, o uso exclusivo para fins científicos, e a possibilidade de solicitar que suas informações sejam tiradas do banco de coleta a qualquer momento sem nenhum prejuízo ao atendimento na unidade de saúde.

Após essas explicações, foi apresentado o termo de consentimento livre e esclarecido, informando qual a sua finalidade e solicitação para assinatura. Conforme a realização dessa etapa, os auxiliares apresentaram os questionários que serviram para coleta de dados. O questionário que embasou a coleta foi criado pelo pesquisador orientador e auxiliares, a partir de questionamentos durante uma disciplina de uma Instituição de Ensino Médico da capital do Estado de Rondônia.

É composto por 15 questões que avaliaram o perfil epidemiológico, se o uso do anticoncepcional oral combinado previne infecções sexualmente transmissíveis, se é necessário o uso de preservativo após iniciar o uso de anticoncepcional oral combinado, por qual motivo se usa preservativo, qual a função da pílula do dia seguinte, se é normal mulher apresentar corrimento vaginal, se toda mulher desenvolve trombose após iniciar o uso de anticoncepcionais hormonais, se ocorre transmissão vertical de sífilis, se a infecção por HPV tem relação com o câncer de colo de útero, se o DIU de cobre pode ser usado por 10 anos, se sentiu angústia ou ansiedade por apresentar suspeita de uma IST, se sente vergonha em buscar atendimento ginecológico e se sabe onde buscar atendimento para realização de preventivo.

Por fim, os dados coletados foram salvos em planilhas no programa Microsoft Office Excel 2013 e Microsoft Word 2013. Dessa forma, foi realizado as análises dos dados, bem como a aplicação destes no próprio programa em que foram gerados. Após estes processos, ocorreu a comparação com a literatura e discussão dos resultados. Este trabalho foi aprovado pelo comitê de ética em pesquisa sob número do CAAE 30646720.0.0000.0013, número do parecer 4.366 .580 em 28 de outubro de 2020.

\section{RESULTADOS}

Durante o estudo foram entrevistadas 46 mulheres, no entanto, apenas 33 se enquadraram nos critérios de inclusão, foram excluídas mulheres que possuíam mais de 40 anos e menores de 18 anos. Com relação à faixa etária das participantes, houve prevalência das idades de 18 a 21 anos, correspondendo a $33 \%$ do total, seguidas pela faixa etária de 30 a 34 anos que corresponde a $21 \%$, a de 35 a 40 anos representando $18 \%$, a de 26 a 29 anos representando $15 \%$ e a de menor percentual, 22 a 25 anos, representando $12 \%$.

Referente aos níveis de escolaridade das participantes, 30\% possuíam o ensino médio completo, $24 \%$ possuíam o ensino fundamental incompleto, $15 \%$ o ensino superior incompleto, $12 \%$ o ensino médio incompleto, $9 \%$ o nível superior completo, $6 \%$ o ensino fundamental completo e $3 \%$ não souberam responder.

Em relação a renda mensal da casa das entrevistadas, $73 \%$ tinham renda equivalente a 1 salário mínimo mensal, 15\% tinham renda de 2 salários mínimos mensais, 9\% não souberam responder, 3\% tinham renda de 3 salários mínimos mensais e nenhuma possuía renda de 4 ou mais salários mínimos mensais.

Ao ser perguntado se a pílula anticoncepcional evita alguma infecção sexualmente transmissível, $79 \%$ afirmaram que não, enquanto $18 \%$ afirmaram que sim, havendo apenas $3 \%$ de respostas em branco. Já em relação a necessidade do uso de preservativos, masculino ou feminino, após início do uso da pílula anticoncepcional, 97\% julgaram ser necessário, 3\% não soube responder e nenhuma afirmou não ser necessário.

Ademais, quando questionadas sobre o motivo pelos quais as mesmas usariam preservativos, as respostas obtidas foram variadas, porém, similares (Quadro 1). 
Quadro 1 - Motivo do uso de preservativos segundo as usuárias entrevistadas na Unidade Básica de Saúde.

\begin{tabular}{|c|c|}
\hline Usuárias & Respostas \\
\hline Usuária 1 & Não pegar doença \\
\hline Usuária 2 & Por causa de doença \\
\hline Usuária 3 & Evita gravidez \\
\hline Usuária 4 & Evita doença \\
\hline Usuária 5 & Não engravidar \\
\hline Usuária 6 & Para não contrair doença e engravidar \\
\hline Usuária 7 & Para proteger de doenças \\
\hline Usuária 8 & Evita doença e filho \\
\hline Usuária 9 & Evita DSTS e gravidez \\
\hline Usuária 10 & Para se cuidar \\
\hline Usuária 11 & Para evitar doença \\
\hline Usuária 12 & Para evitar doença e filho \\
\hline Usuária 13 & Para evitar DST \\
\hline Usuária 14 & Para evitar doença e ter filhos \\
\hline Usuária 15 & Para evitar doença e gravidez \\
\hline Usuária 16 & Por causa de doença sexualmente transmissível porque a pílula evita só de ter filhos \\
\hline Usuária 17 & Para prevenir doença e gravidez indesejada \\
\hline Usuária 18 & Para evitar transmissão de doença \\
\hline Usuária 19 & Prevenção \\
\hline Usuária 20 & Para evitar doença e gravidez indesejada \\
\hline Usuária 21 & Evitar doença sexualmente transmissível \\
\hline Usuária 22 & Evita de contrair doença e evitar uma gravidez indesejada \\
\hline Usuária 23 & Para não pegar doença e não engravidar \\
\hline Usuária 24 & Prevenir doença \\
\hline Usuária 25 & Para se prevenir das doenças \\
\hline Usuária 26 & Evita uma gravidez indesejada e evita doença sexualmente transmissível \\
\hline Usuária 27 & Para não pegar doença sexual \\
\hline Usuária 28 & Não pegar doença sexualmente transmissível \\
\hline Usuária 29 & Para evitar doença e gravidez \\
\hline Usuária 30 & Evitar doença e filhos \\
\hline Usuária 31 & Prevenir DST \\
\hline Usuária 32 & Prevenir doença \\
\hline Usuária 33 & Prevenir doença \\
\hline
\end{tabular}

Fonte: SILVA ACR, et al., 2021.

Ao serem interrogadas sobre os conhecimentos acerca da pílula do dia seguinte e a sua função, foram levantadas as seguintes respostas (Quadro 2). 
Quadro 2 - Função da pílula do dia seguinte segundo as usuárias entrevistadas na Unidade Básica de Saúde.

\begin{tabular}{|c|c|}
\hline Usuárias & Respostas \\
\hline Usuária 1 & Não engravidar \\
\hline Usuária 2 & Evitar gestação \\
\hline Usuária 3 & Abortiva \\
\hline Usuária 4 & Evita gravidez \\
\hline Usuária 5 & Não sei \\
\hline Usuária 6 & Se fizer alguma besteira não engravidar \\
\hline Usuária 7 & Para prevenir gravidez \\
\hline Usuária 8 & Não sei \\
\hline Usuária 9 & Evitar a concepção \\
\hline Usuária 10 & Para não ter criança \\
\hline Usuária 11 & Para evitar filho \\
\hline Usuária 12 & Acho que para não engravidar \\
\hline Usuária 13 & Evita fecundação do espermatozoide no ovário \\
\hline Usuária 14 & Não sei \\
\hline Usuária 15 & Não sei \\
\hline Usuária 16 & Para não ter filhos \\
\hline Usuária 17 & Para não engravidar \\
\hline Usuária 18 & Para prevenir de não engravidar \\
\hline Usuária 19 & Evita gravidez \\
\hline Usuária 20 & Evitar gravidez indesejada \\
\hline Usuária 21 & Evitar gravidez indesejada \\
\hline Usuária 22 & Evita gravidez quando ocorre relação sem proteção \\
\hline Usuária 23 & Para evitar gravidez \\
\hline Usuária 24 & Não sei \\
\hline Usuária 25 & Evita gravidez \\
\hline Usuária 26 & Não sei, porém, acredito que é para evitar gravidez \\
\hline Usuária 27 & Evita a fecundação do esperma \\
\hline Usuária 28 & Quando não usa camisinha e pode ter engravidado a mulher toma para não engravidar \\
\hline Usuária 29 & Evitar \\
\hline Usuária 30 & Evitar gravidez indesejada, parar o ciclo \\
\hline Usuária 31 & Evitar gravidez \\
\hline Usuária 32 & Evitar gravidez \\
\hline Usuária 33 & Não sei \\
\hline
\end{tabular}

Fonte: SILVA ACR, et al., 2021.

Com referência a ocorrência de corrimento vaginal, as usuárias foram questionadas sobre a possibilidade deste ocorrido ser normal em mulheres, tendo $79 \%$ respondido que não, $18 \%$ respondido que sim e $3 \%$ que sim e não, com $0 \%$ de abstenção de resposta. Ao serem indagadas sobre a possibilidade de toda mulher que faz uso de anticoncepcional desenvolver trombose, $48 \%$ responderam que não, $36 \%$ responderam que sim, $9 \%$ não tiveram resposta e apenas $6 \%$ não disseram não saber.

Quanto à possibilidade de uma mulher grávida portadora de sífilis transmitir a doença para a criança durante a gestação, $91 \%$ julgaram ser possível, $6 \%$ afirmaram que não, $3 \%$ responderam sim/não e nenhuma não soube responder. Já em relação ao HPV, foi-se perguntado se há relação do mesmo com o desenvolvimento do câncer de colo do útero, na qual $67 \%$ responderam que sim, $24 \%$ responderam que não e $9 \%$ não tiveram resposta. 
Para caracterizar o conhecimento das participantes sobre o DIU de cobre, foi perguntado se elas julgavam verdadeira a seguinte afirmação "O DIU de cobre é um método que pode ser usado por 10 anos, sendo necessário apenas consultas regulares para acompanhamento", tendo $70 \%$ respondido que sim e $30 \%$ respondido que não. Ao ser perguntado se as entrevistadas já se sentiram ansiosas ou angustiadas por apresentar algum sintoma que suspeitou ser uma infecção sexualmente transmissível, $55 \%$ responderam que sim e $45 \%$ responderam que não.

Por outro lado, quando questionadas se sentiam vergonha de buscar atendimento em um ambulatório de ginecologia, $88 \%$ responderam que não e apenas $12 \%$ afirmaram se sentir envergonhadas. Por fim, ao serem indagadas sobre o conhecimento de locais para busca de atendimento e realização do exame preventivo, $97 \%$ afirmaram saber onde procurar atendimento e apenas 3\% afirmaram não saber.

\section{DISCUSSÃO}

O levantamento de dados do presente estudo, indicou a prevalência de $33 \%$ na faixa etária de 18 a 21 anos, quando comparamos tais dados ao estudo realizado por Silva ACR, et al. (2020) em outra unidade básica de saúde também situada na capital do Estado de Rondônia, encontramos similaridade entre as faixas etárias mais prevalentes, com predomínio de $25,64 \%$ com idade entre 18 a 23 anos.

Já em outro estudo proposto por Silva ACR, et al. (2019), com levantamento de dados em outra unidade básica de saúde da cidade de Porto Velho, houve coincidência entre o nível de escolaridade predominante, tendo $41 \%$ das usuárias concluído o ensino médio e já no presente estudo com resultado de $30 \%$ do espaço amostral. No que tange a renda média, tanto no estudo realizado por Silva ACR, et al. (2019) e (2020) predominou em $52 \%$ e $35,9 \%$ respectivamente o valor mensal de 1 salário mínimo, indo ao encontro dos dados encontrados na atual pesquisa.

Esse cenário, demonstra que as parcela de usuárias são de baixa renda o que se relaciona diretamente ao chamado de causalidade entre renda e saúde, visto que, um nível maior de renda permite usufruir de melhores condições de saúde (SANTOS AMA, et al., 2012). Assim, nota-se a importância do Sistema Único de Saúde para tais mulheres, uma vez que é o local de acesso ao atendimento à população que é pautado na disponibilidade de atenção à saúde, acessibilidade aos cuidados de saúde, aceitabilidade e qualidade (DANTAS MNP, et al., 2021).

Diante do exposto, notamos que mesmo situadas em áreas geográficas distintas o público-alvo das UBS de Porto Velho possui em suma maioria mulheres que podem encontrar-se em vulnerabilidade econômica e social. Além disso, com a maior parte das participantes em idade reprodutiva, suscita-se maior taxa de gravidez e maternidade, estando relacionado também a maior taxa de práticas a relações sexuais (MOURA LNB, et al., 2014).

A partir desse ponto, é necessárias políticas públicas que orientam e ofertam medidas educativas e acesso a métodos contraceptivos de alta eficácia, contornando as práticas influenciada apenas pela abstinência sexual, uma vez que, a literatura traz que esse método apenas pode retardar em dois anos o início da vida sexual, porém quando ocorre é há maiores chance de não haver o uso de contraceptivos indicando aumento do risco para transmissão de IST ou gravidez (CABRAL CS e BRANDÃO ER, 2020).

Diante desse cenário, é importante o conhecimento acerca dos métodos contraceptivos e IST. Com base nisso, as respostas acerca se as pílulas anticoncepcional evita IST foi satisfatória, visto que $79 \%$ afirmaram que tais pílulas não evitam a transmissão de IST e 97\% informaram que é necessário o uso de preservativo concomitante ao uso dos anticoncepcionais orais para prevenção de doenças. O uso desses dois métodos contraceptivos foram descritos como os mais prevalentes, e o uso combinado o segundo mais frequente entre jovens conforme o estudo realizado por Silva AF e Lopes MHBM (2018).

Quando questionado o motivo do uso de preservativo houve variadas respostas, mas todas satisfatórias quanto a finalidade do uso, no entanto, o estudo feito por Delatorre MZ e Dias ACG (2015) discute que nem sempre o conhecimento adequado significa o uso adequado e práticas contraceptivas seguras, principalmente entre jovens de 15 a 24 anos, estando relacionado a faixa etária prevalente do presente estudo. 
Ao passo que pode ocorrer do uso não adequado de preservativo, entender sobre contracepção de emergência se faz importante, seguindo esse pressuposto ao questionar o conhecimento acerca de tal método, 7 das 33 participantes não sabiam a finalidade e 1 informou ser um método abortivo. No estudo feito Lacerda JOS, et al. (2019), descreveu que a informação sobre contracepção de emergência em mulheres jovens é mais prevalente naquelas em período escolar ou com ensino médio completo, indo ao encontro da escolaridade prevalente no presente estudo e consoante a maioria que compreendia a função da pílula do dia seguinte.

Além desses métodos contraceptivos foi questionado acerca do dispositivo intrauterino de cobre (DIU), as respostas foram satisfatórias para o tempo de uso do método, tendo $70 \%$ respondido adequadamente, esse método contraceptivo está entre os mais usados em todo o mundo, além de apresentar taxas de falhas muito baixas (HOLANDA AAR, et al., 2012).

Avaliando o conhecimento acerca do HPV, 67\% sabiam da relação desse vírus com o câncer de colo de útero e $97 \%$ informaram conhecer quais lugares realizam atendimento para coleta de preventivo. Tais dados se demonstram satisfatório apesar de ainda notar no Brasil baixa adesão a realização do exame que pode ser motivado por vergonha, o que vai de encontro aos dados obtidos no levantamento, visto que $88 \%$ responderam que não sentem vergonha em buscar atendimento ginecológico (SILVA JN, et al., 2020).

O resultado satisfatório quanto o conhecimento acerca da transmissão vertical de sífilis promove conscientização quanto as complicações dessa doença durante a gestação, já que, a sífilis afeta um elevado número de gestante com alto índice de desfecho negativo como óbito fetal, prematuridade, baixo peso ao nascer ou recém-natos infectados (DOMINGUES RMSM e LEAL MD, 2016).

\section{CONCLUSÃO}

Os resultados evidenciam que a maioria das usuárias estão na faixa etária de 18 a 21 anos, em que a maioria possui o Ensino Médio completo, e vivem em uma situação de vulnerabilidade econômica visto que a renda mensal de $75 \%$ das entrevistadas é de 1 salário mínimo. Observou-se resultados satisfatórios quando questionadas se métodos anticoncepcionais evitariam doenças sexualmente transmissíveis e também a respeito do uso combinado de anticonceptivos orais e preservativos, porém ainda há necessidade de ser realizado maior promoção e prevenção com esses temas. De acordo com os dados $18 \%$ afirmaram que anticonceptivos evita sim IST's além de 7 entrevistadas não obtinham conhecimento da finalidade de métodos anticonceptivos, 1 afirmou ser um método abortivo.

\section{REFERÊNCIAS}

1. BRITTO AMA, et al. Detecção de infecções sexualmente transmissíveis em um centro ginecológico brasileiro: alta prevalência de coinfecções. Jornal Brasileiro de Patologia e Medicina Laboratorial, 2018; 54 (6): 393-400.

2. CABRAL CS, BRANDÃO ER. Gravidez na adolescência, iniciação sexual e gênero: perspectivas em disputa. Cad. Saúde Pública, 2020; 36(8): 1-5.

3. CARRENO I, et al. Uso de métodos contraceptivos entre mulheres com vida sexual ativa em São Leopoldo, Rio Grande do Sul, Brasil. Cadernos de Saúde Pública, 2006; 22(5): 1101-1109.

4. CURITIBA. Secretaria Municipal de Saúde. Planejamento familiar, 2002.

5. CURITIBA. Secretaria Municipal de Saúde. Protocolo de atenção à saúde do adolescente, 2006.

6. DANTAS MNP, et al. Fatores associados ao acesso precário aos serviços de saúde no Brasil. Rev Bras Epidemiol, 2021; 24(21): 1-13.

7. DELATORRE MZ, DIAS ACG. Conhecimentos e práticas sobre métodos contraceptivos em estudantes universitários. Revista da SPAGESP, 2015; 16(1): 60-73.

8. DEL BIANCO AAM. Nas trilhas das DST e HIV com os adolescentes e jovens: um projeto de intervenção em educação e saúde em uma escola pública de ensino fundamental de Santana do Araguaia/PA. Trabalho de Conclusão de Curso. (Especialização em Gestão da Política de DST, AIDS, Hepatites Virais e Tuberculose Educação a Distância) - Universidade Federal do Rio Grande do Norte, Natal, 2017.

9. DOMINGUES RMSM, LEAL MC. Incidência de sífilis congênita e fatores associados à transmissão vertical da sífilis: dados do estudo Nascer no Brasil. Cadernos de Saúde Pública, 2016; 32(6): e00082415. 
10. DUARTE MTC, et al. Vulnerabilidade de mulheres vivendo com HIV/Aids. Revista Latino-Americana de Enfermagem, Ribeirão Preto, 2014; 22(1): 68-75.

11. HOLANDA AAR, et al. Controvérsias acerca do dispositivo intrauterino: uma revisão. Feminina, 2012; 41(3): 1-6.

12. LACERDA JOS, et al. O Uso Indiscriminado da Anticoncepção de Emergência: Uma Revisão Sistemática da Literatura. Id On Line Revista Multidisciplinar e de Psicologia,2019; 13(43): 379-386.

13. LARA LAS; ABDO CHN. Aspectos da atividade sexual precoce. Rev Bras Ginecol Obste, 2015; 37(5): $199-202$.

14. LUPPI CG, et al. Diagnóstico precoce e os fatores associados às infecções sexualmente transmissíveis em mulheres atendidas na atenção primária. Revista Brasileira de Epidemiologia, 2011;14(3), 467-477.

15. MOURA LNB, et al. Multiparidade entre adolescentes e jovens e fatores de risco em Teresina/Piauí. Revista Adolescência e Saúde, Rio de Janeiro, 2014; 11(3): 51-62.

16. NEVES RG, et al. Simultaneidade de comportamentos de risco para infecções sexualmente transmissíveis em adolescentes brasileiros, 2012. Epidemiol. Serv. Saude, 2017; 26(3): 443-454.

17. OSIS MJMD. Atenção integral a saúde da mulher, o conceito e o programa: história de uma intervenção. Dissertação (Mestrado) - Universidade Estadual de Campinas, Instituto de Filosofia e Ciências Humanas, Campinas, São Paulo, 1994.

18. PINTO VM, et al. Fatores associados às infecções sexualmente transmissíveis: inquérito populacional no município de São Paulo, Brasil. Ciência \& Saúde Coletiva, 2018; 23(7): 2423-2432.

19. RASMUSSEN VS, et al. Conhecimento e uso prévio de métodos anticoncepcionais em gestantes adolescentes. Revista Arquivos Catarinenses de Medicina, 2011; 40(4): 52-57.

20. RONDÔNIA (Estado). Decreto $n^{\circ} 24.871$, de 16 de março de 2020. Decreta situação de emergência no âmbito da Saúde Pública do Estado e dispõe sobre medidas temporárias de prevenção ao contágio e enfrentamento da propagação do COVID-19. Diário Oficial do Estado de Rondônia, 2020.

21. SANTOS AMA, et al. Causalidade entre renda e saúde: uma análise através da abordagem de dados em painel com os estados do Brasil. Estudos Econômicos, São Paulo, 2012; 42(2): 229-26

22. SAITO MI, LEAL MM. Educação sexual na escola. Revista Pediatria, 2000; 22(1): 44-48.

23. SILVA ACR, et al. Análise do conhecimento de pacientes e profissionais de saúde de uma unidade de saúde da família de Porto Velho - Rondônia - acerca do aleitamento materno. Saber Científico, 2020; 9(1):121-129.

24. SILVA ACR, et al. Importância do pré-natal na opinião das usuárias de uma unidade básica de saúde da família em Porto Velho, Rondônia. Saber Científico, 2019; 8(2): 89-98.

25. SILVA AF, LOPES MHBM. Uso de métodos anticoncepcionais entre adolescentes de ensino médio. Biblioteca Virtual em Saúde, 2018; 15(2): 102-112.

26. SILVA JN, et al. Exame de Papanicolau: conhecimentos de mulheres em uma unidade de saúde escola da Amazônia ocidental. Revista Eletrônica Acervo Saúde, 2020; 12(7): 1-10.

27. TERRA AAA, SILVA GA. Representando as ações preventivas das IST/Aids realizadas por enfermeiros na atenção básica. Enfermagem Brasil, 2017; 16(5): 1-8. 